Pacific Journal of Mathematics

NORMAL FORM FOR A PFAFFIAN 


\section{NORMAL FORM FOR A PFAFFIAN}

\section{RICHARD ARENS}

1. Introduction. It is well known that "generally" (which is to is the say, usually) a Pfaffian, or 1-form,

$$
\alpha=a_{1}(x) d x^{1}+\cdots+a_{n}(x) d x^{n}
$$

in $\boldsymbol{R}^{n}$ has one or the other of the two representations

$$
\alpha=u^{1} d u^{2}+\cdots u^{2 p-1} d u^{2 p}+\left\{\begin{array}{l}
0 \\
d u^{2 p+1}
\end{array}\right.
$$

in an appropriate coordinate system $\left(u^{1}, u^{2}, \cdots, u^{n}\right)$. Moreover, the last index $(2 p$ or $2 p+1)$ appearing in 1.1 is the rank $r$ of the $n \times(n+1)$ matrix

$$
\left[\begin{array}{cccc}
a_{1} & a_{2} & \cdots & a_{n} \\
a_{11} & a_{12} & \cdots & a_{1 n} \\
a_{21} & a_{22} & \cdots & x_{2 n} \\
\cdot & & \\
a_{n 1} & a_{n 2} & \cdots & a_{n n}
\end{array}\right],
$$

in which $a_{i j}$ is an abbreviation for $\partial a^{i} / \partial x^{i}-\partial a_{j} / \partial x^{i}$.

It goes without saying that this is regarded as a local proposition, indicating that if the rank of 1.2 were constant in some neighborhood of a point $\boldsymbol{P}_{0}$, then a smaller neighborhood of $\boldsymbol{P}_{0}$ and a curvilinear coordinate system valid on that neighborhood, could be found yielding the representation 1.1 .

It is very probable that a satisfactory proof concerning the possibility of reducing a Pfaffian in this way exists in the literature ${ }^{1}$. Nevertheless, it should be pointed out that the accepted version is not exactly true (and this is part of our object in writing this paper.)

Consider the Pfaffian $y d x+2 x d y$ in ordinary $\boldsymbol{R}^{2}$. The Pfaffian matrix is

$$
\left[\begin{array}{rr}
y & 2 x \\
0 & -1 \\
1 & 0
\end{array}\right] \text {. }
$$

Received June 7. 1963.

1 For references to the older literature, see pp. 324-6 of E. Weber's article in the Encyklop. d. Math. Wiss. Band II, Erster Teil, Erste Hälfte (1.I) Teubner (1916). This article attributes to Frobenius a proof of the sort of proposition stated above, which we will therefore call the accepted version. 
It has rank 2 everywhere. Indeed, this Pfaffian can be written

$$
y^{-1} d\left(x y^{2}\right) \text {, }
$$

but this does not confirm the accepted version for points at which $y=0$. (Actually, as our Theorem 2.1 shows, the representation $u d v$ is possible at any point different from the origin.) We will now indicate why there do not exist functions $u$ and $v$ each $\mathscr{C}^{\infty}$ in a neighborhood of $(0,0)$ such that $y d x+2 x d y=u d v$. It is evident that $v=f\left(x y^{2}\right)$, whence $f(\lambda)=v\left(\lambda \varepsilon^{-2}, \varepsilon\right)$ for some $\varepsilon \neq 0$. This shows that $f$ is $\mathscr{C}^{\infty}$ in some neighborhood of 0 . From this we obtain $y^{-1} d\left(x y^{2}\right)=y d x+2 x d y=u f^{\prime}\left(x y^{2}\right) d\left(x y^{2}\right)$ wherever $y \neq 0$, or $y^{-1}=$ $u f^{\prime}\left(x y^{2}\right)$ where $y \neq 0$, which shows that $y^{-1}$ is bounded where $y \neq 0$ an absurdity. Thus the accepted version is defective.

The only explanation of this state of affairs is that Pfaff's problem problem is, by the authors mentioned in our bibliography at least, not regarded as an "advanced calculus" type of problem (or theorem.) This is made explicit by Thomas in his postulational approach; and it is made evident by the fact that a reading of the first line of his table on p. 45, shows that $\omega=y d x+2 x d y$ should have a canonical form $p d q$, since $0 \neq d \omega=d x \wedge d y$ and $\omega \wedge d \omega=0$, if that theory really did apply.

Our proof, given below, shows that a sufficient additional assumption is that the Pfaffian does not vanish at $\boldsymbol{P}$ (and this is already implicitly assumed when $r$ is odd.)

A person might imagine that it would be an easy task to glance at some rather elementary proof such as the first proof presented by Gours at in his book, and verify that the denominator of each quotient formed by Goursat does not vanish at $\boldsymbol{P}$ if the Pfaffian does not. But this proof is by induction, and it is apparent that if you lop terms off a Pfaffian you may find at some lower (even) dimension that the non-vanishing feature has been lost. The exercise of vigilance of this kind almost doubles the length of Goursat's proof. On the other hand, explicit use of Frobenius' theorem on involutory vector field systems, enables us to present a proof which is shorter than Goursat's.

Cartan, in his book (p. 57) sketches a theory of Pfaffian equations, which is to say that two Pfaffians differing by a factor which is only a function are regarded as equivalent. It appears that he permits these functions to have zeros. (Indeed if he did not, then his short proof on p. 57 would establish the accepted version).

2. The reduction of Pfaffians. The solution of "Pfaff's Problem" lies in the following theorem.

2.1 Theorem. Let $\alpha=a_{1} d x^{1}+\cdots+a_{n} d x^{n}$ be a Pfaffian with 
$\mathscr{C}_{\infty}$ components $a_{1}, \cdots, a_{n}$ defined in the neighborhood of the origin 0 in $\boldsymbol{R}^{n}$. Let

$$
a_{i j}=\frac{\partial a_{i}}{\partial x^{j}}-\frac{\partial a_{j}}{\partial x^{i}},
$$

and suppose that the rank of the matrix

2.11

$$
\left[\begin{array}{ccc}
a_{1} & \cdots & a_{n} \\
a_{11} & \cdots & a_{1 n} \\
\cdot & & \cdot \\
\cdot & & \cdot \\
\cdot & & \cdot \\
a_{n 1} & \cdots & a_{n n}
\end{array}\right]
$$

is constant in a neighborhood of 0 . Let that constant be $r$. If $r$ is even,

2.12 suppose that the $a_{1}, \cdots, a_{n}$ do not all vanish at 0 .

Then there is a coordinate system $\left(u^{1}, \cdots, u^{n}\right)$ defined in $a$ neighborhood of 0 in $\boldsymbol{R}^{n}$ such that

2.13

$$
\alpha=u^{1} d u^{2}+\cdots+u^{2 p-1} d u^{2 p}+ \begin{cases}0 & \text { if } r=2 p \\ d u^{2 p+1} & \text { if } r=2 p+1 .\end{cases}
$$

We observe first some invariance properties of the rank of 2.11.

2.2 Prop. Let $\boldsymbol{P}$ be a point of $\boldsymbol{R}^{n}$ and let cls $(d, \boldsymbol{P})$ be the class of $\alpha$ at $\boldsymbol{P}$, namely the linear dimension of the class of vectors $X$ at $\boldsymbol{P}$ such that

2.21

$$
\langle\alpha, X\rangle=0
$$

and

2.22

$$
\langle d \alpha ; X, Y\rangle=0 \text { for all vectors } Y \text { at } \boldsymbol{P} \text {. }
$$

Then cls $(\alpha, \boldsymbol{P})$ equals the rank of 2.11 at $\boldsymbol{P}$.

The proof of 2.2 is by simple linear algebra.

Thus cls $(\alpha, P)$ may be calculated as the rank of

2.23

$$
\left[\begin{array}{cc}
b_{1}, \cdots, b_{n} \\
b_{11}, \cdots, b_{1 n} \\
\cdot & \cdot \\
\cdot & \\
b_{n 1}, \cdots, & \cdot
\end{array}\right]
$$


where $\alpha=b_{1} d y^{1}+\cdots+b_{n} d y^{n}$ in some other coordinate system, and $b_{i j}=\left(\partial b_{i} / \partial y^{j}\right)-\left(\partial b_{j} / \partial y^{i}\right)$.

Let $\alpha^{(1)}=\alpha, \alpha^{(2)}=d \alpha, \alpha^{(3)}=\alpha \wedge d \alpha, \alpha^{(4)}=d \alpha \wedge d \alpha, \alpha^{(5)}=\alpha \wedge d \alpha, \cdots$ etc.

2.24 Prop. (cf. Cartan, p. 58). The class of $\alpha$ at a point $\boldsymbol{P}$ is the greatest integer $r$ for which $\alpha^{(r)}(\boldsymbol{P}) \neq 0$.

Proof. Suppose $\boldsymbol{P}$ is the origin, which we shall denote by $0 . \quad$ A theorem of linear algebra [cf. Cartan, p. 12] shows that a coordinate system $\left(y^{1}, \cdots, y^{n}\right)$ can be found such that $d \alpha$ at the origin has the form $d y^{1} \wedge d y^{2}+\cdots+d y^{2 p-1} \wedge d y^{2 p}$. In this coordinate system, $\alpha$ has components $b_{1}, \cdots, b_{n}$, and

$$
d \alpha=\sum_{i, j} \frac{\partial b_{i}}{\partial y^{j}} d y^{j} \wedge d y^{i}=\frac{1}{2} \sum_{i, j} b_{i j} d y^{j} \wedge d y^{i} .
$$

The $n$-by- $n$ array of the $b_{i j}(0)$ is thus as follows:

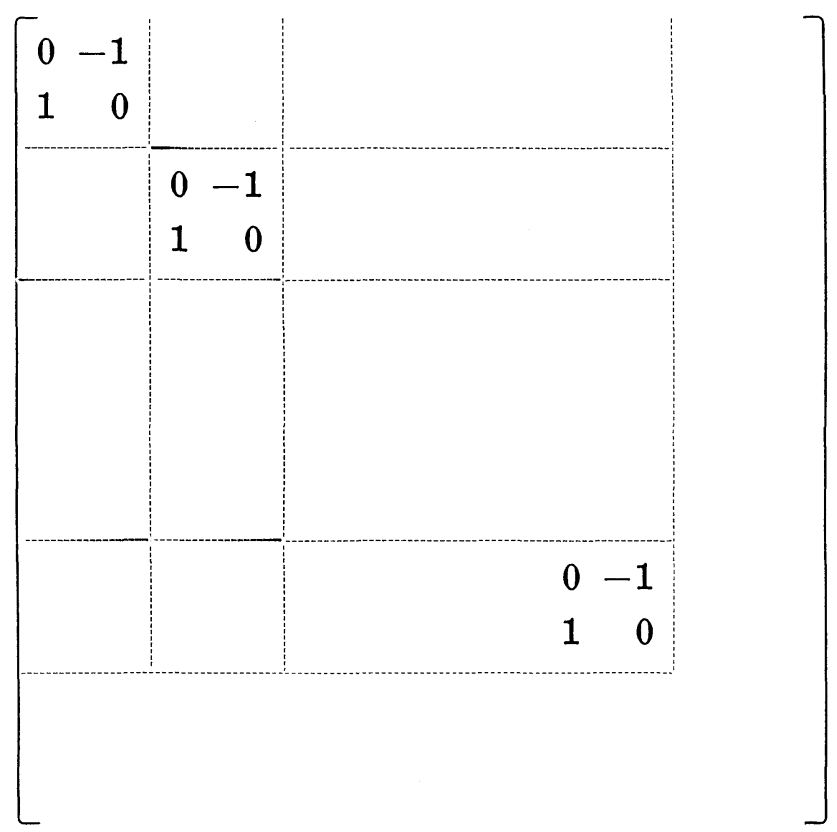

with $p$ such boxes situated on the diagonal (note $2 p \leqq n$ ) and zeros in the unmarked places. This array has rank $2 p$. Considering the way in which it enters into 2.23 we see that 2.23 has rank $2 p$ precisely if $g_{2 p+1}(0), b_{2 p+2}(0), \cdots, b_{n}(0)$ are 0 , and rank $2 p+1$ otherwise.

Returning to the form of $d \alpha$ at 0 , we see that $\alpha^{(t)}(0)=0$ for $t>2 p+2$. Indeed,

$$
\alpha^{(2 p+1)}(0)=\left[b_{2 p+1}(0) d y^{2 p+1}+\cdots+b_{n}(0) d y^{n}\right] \wedge d y^{1} \wedge d y^{2} \wedge \cdots d y^{2 p} .
$$


This is 0 or non- 0 according to whether $b_{2 p+1}(0)=\cdots=b_{n}(0)=0$, or not. This proves 2.24 .

We will now prove 2.2 by induction, assuming it true for each Pfaffian $\beta$ whose class is less than $r$. We suppose, then, that $\alpha=$ $a_{i} d x^{i}$ (using the summation convention in this proof) is of class $r$ in a neighborhood $U$ of 0 , in $\boldsymbol{R}^{n}$.

The constancy of cls $(\alpha,$.$) in a neighborhood V$ of 0 enables one to find $n-r$ vector fields $X_{r+1}, \cdots X_{n}$ defined on a neighborhood $W$ of 0 such that at each point $\boldsymbol{P}$ of $W$, any vector $X$ such that 2.21 and 2.22 hold, is representable in the form

$$
\lambda^{r+1} X_{r+1}(\boldsymbol{P})+\cdots+\lambda^{n} X_{n}(\boldsymbol{P}) .
$$

Let sing-sol $(\alpha, 0)$ designate the class of vector fields $X$ representable in the form

$$
f^{r+1} X_{r+1}+\cdots+f^{n} X_{n}
$$

where $f^{r+1}, \cdots, f^{n}$ are $\mathscr{C}^{\infty}$ functions on some neighborhood of 0 . (We will stop naming these neighborhoods). The term sing-sol refers to the fact that these vectors are both solvers of $\alpha(2.21)$ and singular for $d \alpha(2.22)$. It is easy to see that if $X$ and $Y$ are vector fields in sing-sol $(\alpha, 0)$ then so is $[X, Y]$. Accordingly, we have here an involutory distribution so that one may assert [cf. Chevalley, p. 89. The theorem holds equally well in the $\mathscr{C}^{\infty}$ situation. Incidentally, 2.1 holds equally well in the analytic situation.] that there is a coordinate system $y^{1}, \cdots, y_{n}$ in a neighborhood of 0 such that sing-sol $(\alpha, 0)$ is generated by the vector fields

2.25

$$
\frac{\partial}{\partial y^{r+1}}, \frac{\partial}{\partial y^{r+2}}, \cdots, \frac{\partial}{\partial y^{n}} .
$$

Let $\alpha=b_{1} d y^{1}+\cdots+b_{n} d y^{n}$. Let $X=\partial / \partial y^{s}$ where $s>r$. Then 2.21 holds and tells us that

2.26

$$
b_{s}=0 \text { for } s>n \text {. }
$$

Let $Y=\partial / \partial y^{i}$. Thus 2.22 holds. Thus

$$
\begin{aligned}
0 & =\left\langle d b_{k} \wedge d y^{k} ; X, Y\right\rangle=\left|\begin{array}{ll}
X\left(b_{k}\right) & X\left(y^{k}\right) \\
Y\left(b_{k}\right) & Y\left(y^{k}\right)
\end{array}\right| \\
& =\frac{\partial b_{k}}{\partial y^{s}} \delta_{i}^{k}-\frac{\partial b_{k}}{\partial y^{i}} \delta_{s}^{k}=\frac{\partial b_{i}}{\partial y^{s}}-\frac{\partial b_{s}}{\partial y^{i}}=\frac{\partial b_{i}}{\partial y^{s}} .
\end{aligned}
$$

Therefore

$$
\alpha=b_{1} d y^{1}+\cdots+b_{r} d y^{r}
$$

where $b_{1}, \cdots, b_{r}$ depend only on $y^{1}, \cdots, y^{r}$. 
Our reduction problem is thus obviously reduced to the case $n=r$. We therefore start all over again, supposing $n=r$.

We consider first the case in which the skew symmetric matrix, the $r$ by $r$ minor of 2.11

$$
\left[a_{i j}\right]_{i, j=1, \cdots, r}
$$

has a nonzero determinant. Then $r$ is necessarily even: $r=2 p$. One can then solve the equations

$$
a_{i j} Y^{j}=a_{i} \quad \text { (summation convention!) }
$$

for the functions $Y^{1}, \cdots, Y^{r}$ which are not all 0 by 2.12. Let $Y$ be the vector field

$$
Y^{j} \frac{\partial}{\partial x^{j}}
$$

There is a coordinate system $y^{1}, \cdots, y^{r}$ such that $Y=\partial / \partial y^{r}$ [cf. Chevalley, p. 89, Lemma 1].

Using the original coordinates, it is easy to see from 2.28 , that for our $Y$

2.29

$$
\langle d \alpha, Y, Z\rangle=\langle\alpha, Z\rangle \text { for every } Z \text {. }
$$

Let $Z=\partial / \partial y^{i}$. Expanding 2.29 (as in the lines between 2.26 and 2.27 above) one obtains

$$
b_{i}=\frac{\partial b_{i}}{\partial y^{r}}-\frac{\partial b_{r}}{\partial y^{i}} .
$$

For $i=r$ this says that $b_{r}=0$, and this in turn shows that $\partial / \partial y\left(b_{i} e^{-y}\right)=$ 0 . (Here $y$ is an abbreviation for $y^{r}$.) Thus $\alpha=e^{y} \beta$ where

$$
\beta=h_{1} d y^{1}+\cdots+h_{r-1} d y^{r-1}
$$

and

2.3

$$
h_{1}, \cdots, h_{r-1} \text { depend only on } y^{1}, \cdots, y^{r-1} \text {. }
$$

We will show that $\beta$ satisfies the conditions of 2.1 with $r$ replaced by $r-1$. Since $\alpha=e^{y} \beta$ we have $d \alpha=e^{v}(d y \wedge \beta+d \beta)$. Now $\alpha^{(2 p)}(0) \neq 0$ so, in a neighborhood of 0 ,

$$
0 \neq(d y \wedge \beta+d \beta) \wedge \cdots \wedge(d y \wedge \beta+d \beta) \quad(p \text { factors }),
$$

whence

$$
0 \neq p d y \wedge \beta \wedge d \beta \wedge \cdots \wedge d \beta+d \beta \wedge \cdots \wedge d \beta
$$




$$
0 \neq p d y \wedge \beta^{(2 p-1)}+\beta^{(2 p)} .
$$

Now $\beta^{\langle 2 p\rangle}$ certainly is 0 , because it has $2 p=r$ differentials $d y^{1}, \cdots, d y^{r-1}$ in it, so that there will be repetitions. Thus $\beta^{(r-1)} \neq 0$ in a neighborhood of 0 , while $\beta^{(r)}=0$ as we just observed. Now 2.24 shows that cls $(\beta, 0)=r-1$. Thus 2.1 applies and

$$
\beta=u^{1} d u^{2}+\cdots+d u^{2 p-1}
$$

in some coordinate system $u^{1}, \cdots, u^{n}$. Thus

$$
\alpha=v^{1} d u^{2}+v^{3} d u^{4}+\cdots+v d u^{2 p-1} \cdot
$$

These functions are independent because $\alpha^{(2 p)} \neq 0$. Hence the desired reduction has been achieved.

The remaining case is where the determinant of $\left[a_{i j}\right]$ vanishes at 0 . In this case its rank is less than $r$, but it cannot be less than $r-1$, because the rank of 2.11 is $r$. Hence its rank is $r-1$ and so $r-1$ is $e v e n^{2}$, as the rank of an antisymmetric matrix is even. In particular, the rank of $\left[a_{i j}\right]$ cannot be $r$, so that $\operatorname{det}\left[a_{i j}\right]=0$. However $\left[a_{i j}\right]$ has at least one $r-1$ rowed minor whose determinant is nonzero. It follows that there are functions $Y^{1}, \cdots, Y^{r}$ such that

$$
a_{i} Y^{i}=1 \text { and } a_{i j} Y^{i}=0 .
$$

Let $Y=Y^{j} \partial / \partial x^{j}$. Properties 2.31 translate into

$$
\langle\alpha, Y\rangle=1 \text { and }\langle d \alpha ; Y, Z\rangle=0 \text { for every } Z \text {. }
$$

We now choose coordinates $y^{1}, \cdots, y^{r}$ so that $Y=\partial / \partial y^{r}$. Using 2.32 in the same way as before, we find that

$$
\alpha=b_{1} d y^{1}+\cdots+b_{r} d y^{r}
$$

where

$2.33 b_{r}=1$ and $b_{1}, \cdots, b_{r-1}$ depend only on $y^{1}, \cdots, y^{r-1}$.

Let $b_{1} d y^{1}+\cdots+b_{r-1} d y^{r-1}=\beta$. Then $\alpha=\beta+d y^{r}$. Now $r=2 p+1$ and $0 \neq \alpha^{(r)}=\alpha \wedge \alpha^{(2 p)}=\alpha \wedge \beta^{(2 p)}$. Hence $\beta^{(r-1)} \neq 0$. On the other hand $\beta^{(r)} \equiv 0$. Thus $r-1$ is clearly the rank of the matrix 2.11 for $\beta$. We must, however, verify that $\beta(\boldsymbol{P}) \neq 0$, because $\operatorname{cls}(\beta)$ is even. This might in fact not be true! We can, in such a case write

$$
\alpha=\left(b_{1}+1\right) d y^{1}+\cdots+b_{r-1} d y^{r-1}+d\left(y^{r}-y^{1}\right)=\gamma+d y,
$$

where $y$ stands for $y^{r}-y^{1}$, and the last equality itself defines $\gamma$. Then $\gamma(\boldsymbol{P}) \neq 0$, and otherwise $\gamma$ has all the properties of $\beta$ that have

${ }^{2}$ [cf. Cartan, p. 13] 
already been established for the induction argument. Thus $\beta$ (or $\gamma$ ) has the form $u^{1} d v^{1}+\cdots+u^{p} d v^{p}$ and $\alpha$ can easily be brought into the desired from.

\section{BIBLIOGRAPHY}

1. E. Cartan, Les systèmes différentielles extérieurs, Hermann (1954), Paris.

2. C. Chevalley, Lie Groups, Princeton (1946).

3. E. Goursat, Leçons sur la Problème de Pfaff, Hermann (1922), Paris.

4. J. M. Thomas, Differential Systems, Amer. Math. Soc. Colloq. Publ. vol. XXI (1937).

UNIVERSITY OF CALIFoRNiA, LoS ANGELES 


\section{PACIFIC JOURNAL OF MATHEMATICS}

\section{EDITORS}

Robert Osserman

Stanford University

Stanford, California

M. G. Arsove

University of Washington

Seattle 5 , Washington
J. DugundjI

University of Southern Califorma: Los Angeles 7, California

Lowell J. Paige

University of California

Los Angeles 24, California

\section{ASSOCIATE EDITORS}

E. F. BECKENBACH

B. H. NeumanN

F. WOLF

K. YosIDA

\section{SUPPORTING INSTITUTIONS}

UNIVERSITY OF BRITISH COLUMBIA

CALIFORNIA INSTITUTE OF TECHNOLOGY

UNIVERSITY OF CALIFORNIA

MONTANA STATE UNIVERSITY

UNIVERSITY OF NEVADA

NEW MEXICO STATE UNIVERSITY

OREGON STATE UNIVERSITY

UNIVERSITY OF OREGON

OSAKA UNIVERSITY

UNIVERSITY OF SOUTHERN CALIFORNIA
STANFORD UNIVERSITY

UNIVERSITY OF TOKYO

UNIVERSITY OF UTAH

WASHINGTON STATE UNIVERSITY

UNIVERSITY OF WASHINGTON

AMERICAN MATHEMATICAL SOCIETY CALIFORNIA RESEARCH CORPORATION SPACE TECHNOLOGY LABORATORIES NAVAL ORDNANCE TEST STATION 


\section{Pacific Journal of Mathematics}

\section{Vol. 14, No. 1 \\ May, 1964}

Richard Arens, Normal form for a Pfaffian .........................

Charles Vernon Coffman, Non-linear differential equations on cones in Banach

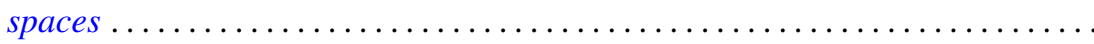

Ralph DeMarr, Order convergence in linear topological spaces ..............

Peter Larkin Duren, On the spectrum of a Toeplitz operator ................

Robert E. Edwards, Endomorphisms of function-spaces which leave stable all

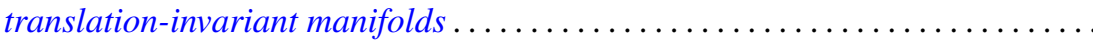

Erik Maurice Ellentuck, Infinite products of isols . . . . . . . . . . . . . . . . 49

William James Firey, Some applications of means of convex bodies . . . . . . . . 53

Haim Gaifman, Concerning measures on Boolean algebras ............. 61

Richard Carl Gilbert, Extremal spectral functions of a symmetric operator. . . . . . 75

Ronald Lewis Graham, On finite sums of reciprocals of distinct nth powers ..... 85

Hwa Suk Hahn, On the relative growth of differences of partition functions ...... 93

Isidore Isaac Hirschman, Jr., Extreme eigen values of Toeplitz forms associated

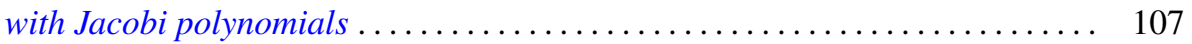

Chen-jung Hsu, Remarks on certain almost product spaces . . . . . . . . . . . 163

George Seth Innis, Jr., Some reproducing kernels for the unit disk . . . . . . . . . 177

Ronald Jacobowitz, Multiplicativity of the local Hilbert symbol . . . . . . . . . . . 187

Paul Joseph Kelly, On some mappings related to graphs ................. 191

William A. Kirk, On curvature of a metric space at a point . . . . . . . . . . . . 195

G. J. Kurowski, On the convergence of semi-discrete analytic functions . . . . . . . 199

Richard George Laatsch, Extensions of subadditive functions . . . . . . . . . . . 209

V. Marić, On some properties of solutions of $\Delta \psi+A\left(r^{2}\right) X \nabla \psi+C\left(r^{2}\right) \psi=0 \ldots 217$

William H. Mills, Polynomials with minimal value sets . . . . . . . . . . . 225

George James Minty, Jr., On the monotonicity of the gradient of a convex

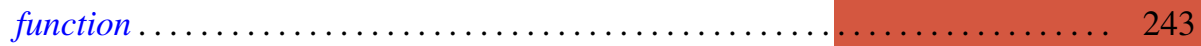

George James Minty, Jr., On the solvability of nonlinear functional equations of 'monotonic' type ................................... 249

J. B. Muskat, On the solvability of $x^{e} \equiv e(\bmod p) \ldots \ldots \ldots \ldots \ldots \ldots \ldots \ldots . \ldots \ldots$

Zeev Nehari, On an inequality of $P . R$. Bessack ................... 261

Raymond Moos Redheffer and Ernst Gabor Straus, Degenerate elliptic

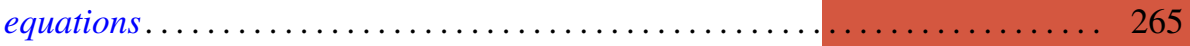

Abraham Robinson, On generalized limits and linear functionals . . . . . . . . . 269

Bernard W. Roos, On a class of singular second order differential equations with a

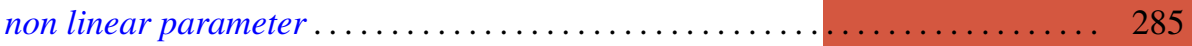

Tôru Saitô, Ordered completely regular semigroups . . . . . . . . . . . . . . . . 295

Edward Silverman, A problem of least area ....................... 309

Robert C. Sine, Spectral decomposition of a class of operators . . . . . . . . . 333

Jonathan Dean Swift, Chains and graphs of Ostrom planes . . . . . . . . . . . 353

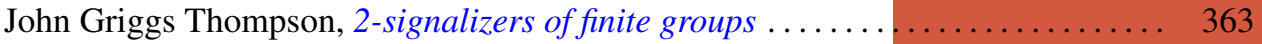

Harold Widom, On the spectrum of a Toeplitz operator . . . . . . . . . . . . . 365 